\title{
A case of primary bilateral breast lymphoma presenting with a painless mass in the left breast
}

\author{
Deniz Esin Tekcan Sanli ${ }^{1,2^{*}}$, Duzgun Yildirim ${ }^{3}$ \\ ${ }^{1}$ DEPARTMENT OF MEDICAL IMAGING TECHNIQUES, VOCATIONAL SCHOOL OF HEALTH SERVICES, ISTANBUL RUMELI UNIVERSITY, ISTANBUL, TURKEY \\ ${ }^{2}$ DEPARTMENT OF RADIOLOGY, ACIBADEM KOZYATAGI HOSPITAL, ISTANBUL, TURKEY \\ ${ }^{3}$ VOCATIONAL SCHOOL OF HEALTH, ACIBADEM UNIVERSITY, ISTANBUL, TURKEY
}

\begin{abstract}
Breast cancer is the most common cancer in women. Patients usually present with a rapidly progressed painless mass in the breast. The first pathological entity to be considered is breast cancer in case of newly developed painless mass, so that radiological and pathological evaluations must be performed. However, some systemic disorders; benign conditions like rheumatological diseases, and also systemic malignancies such as lymphoma, can mimic breast cancer by involving the breast. It is necessary to distinguish between such diseases through a correct diagnosis, because they have very different treatments and prognoses. We present a case diagnosed as bilateral primary breast lymphoma with a palpable mass in a single breast, its clinical and radiological characteristics. This case report suggests that systemic diseases should be considered in the differential diagnosis of breast diseases and breast masses, especially when there is bilaterally involvement, as discussed in this article.
\end{abstract}

\author{
Category: Case Presentation \\ Received: February 09, 2021 \\ Accepted: March 25, 2021 \\ Published: May 10, 2021 \\ Keywords: \\ primary breast lymphoma, bilaterally breast lymphoma, advanced \\ stage, breast implant-associated lymphoma, intramammarian lymph node \\ * Corresponding author: \\ Deniz Esin Tekcan Sanli, \\ Department of Medical Imaging Techniques, Vocational School of \\ Health Services, Istanbul Rumeli University, Istanbul, Turkey \\ E-mail: tekcandenizesin@gmail.com
}

\section{Introduction}

Breast cancer is the most common cancer in women, and its frequency is gradually increasing [1]. Family history of breast cancer, advanced age, hormone therapy, not giving birth, not breast-feeding, are the main known risk factors for breast cancer. The axillary involvement at the time of diagnosis increases the stage, so that it worsens the prognosis and increases the risk of lymphohematogenous spread [1]. Therefore, the condition of the axilla at the time of diagnosis is very important.

Some benign or systemic malignant diseases can mimic breast cancer, with the clinical and imaging changes affecting both breasts and axillary lymph nodes [2]. Usually, invasive breast cancer can be excluded by an adequate history, physical examination, and radiological imaging. In this case report, it is presented a female patient who was admitted to our hospital with a mass in her left breast. There were also bilaterally axillary, inguinal and cervical multiple lymphadenopathies (LAPs). The results of the physical and imaging examination suggested a preliminary diagnosis of lymphoma, while the biopsy and histopathological exam established the diagnosis of bilaterally primary breast lymphoma.

\section{Case report}

A 47-year-old nulliparous female patient, who had been in menopause for 3 years and without radiological followup, was admitted to our hospital with a palpable mass in the left breast. Physical examination of the patient, who had no family history of breast cancer, revealed a soft, painless mass lesion with a diameter of $5 \mathrm{~cm}$ in the upper outer quadrant of the left breast, and bilateral axillary LAPs filling both axillary fossae.

On mammographic examination, a mass opacity with smooth contours was observed in the upper outer quadrant of the left breast, which appears as a heterogeneous dense breast structure (ACR-Type C) (Figure 1). At ultrasonographic evaluation, widespread heterogeneity in both breasts, more prominent in the left breast, hypovascular hypoechoic patchy areas in periareolar/ retroareolar areas, and diffuse thickening in the ductus walls were observed (Figure 2). In the upper outer quadrant of left breast, a mass lesion with smooth lobulated contours, heterogeneous internal structure $50 \times 35 \mathrm{~mm}$ in size, hypoechoic, intense vascularized in color doppler sonography and soft color-coded in elastographic evaluation was observed (Figure 2). 


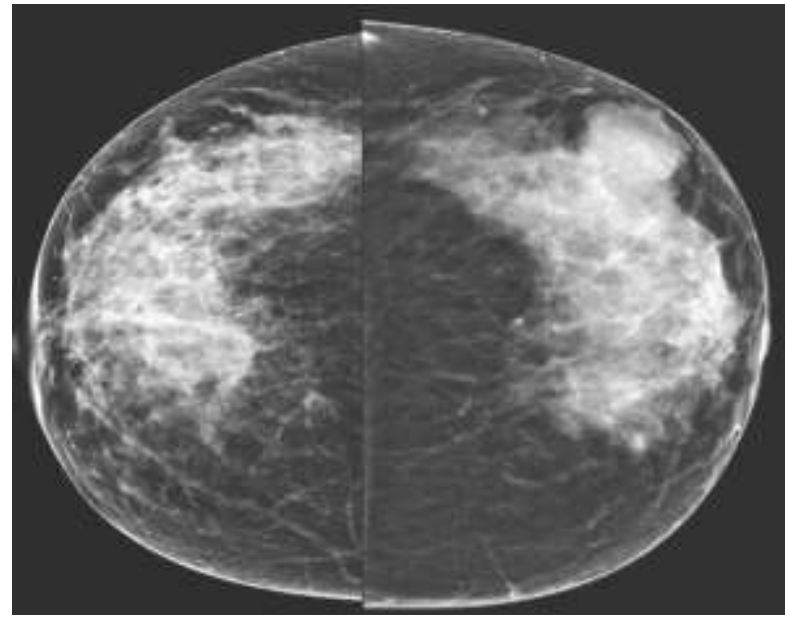

Figure 1a. In the heterogeneous dense breast parenchyma, a mass opacity with smooth lobulated contours in the upper outer quadrant of the left breast can be seen.

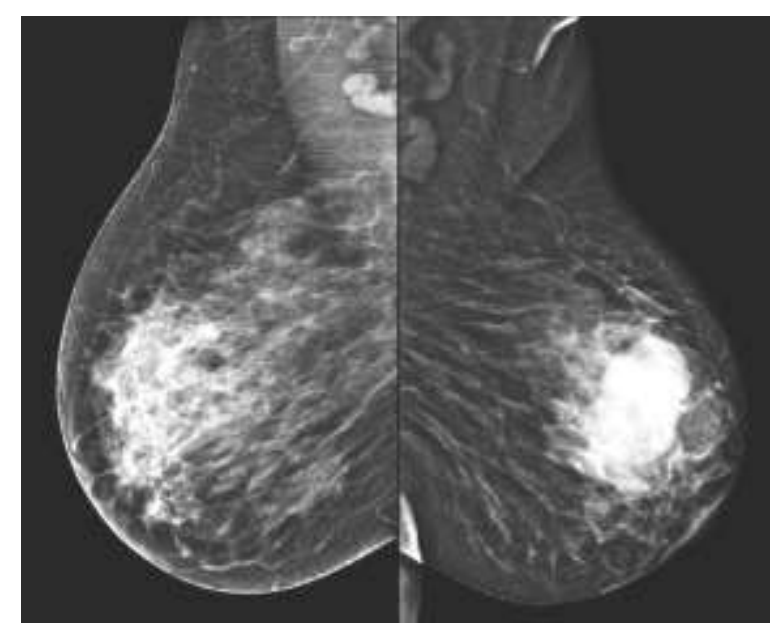

Figure 1b. Note that the mass has a fairly smooth contour, and also note the bilateral axillary LAPs.

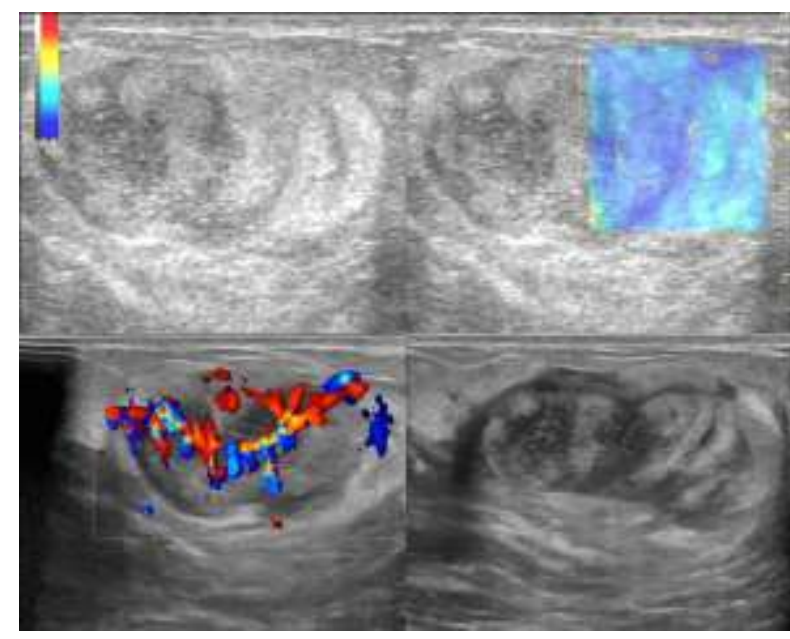

Figure 2a. Ultrasonographic image of the palpable mass in the left breast (gray scale-dopplerelastography, respectively). The mass lesion in heterogeneous internal structure with smooth lobulated contours shows significant internal blood supply in Doppler sonography, but unlike other malignancies, it shows soft color coding elastographically.

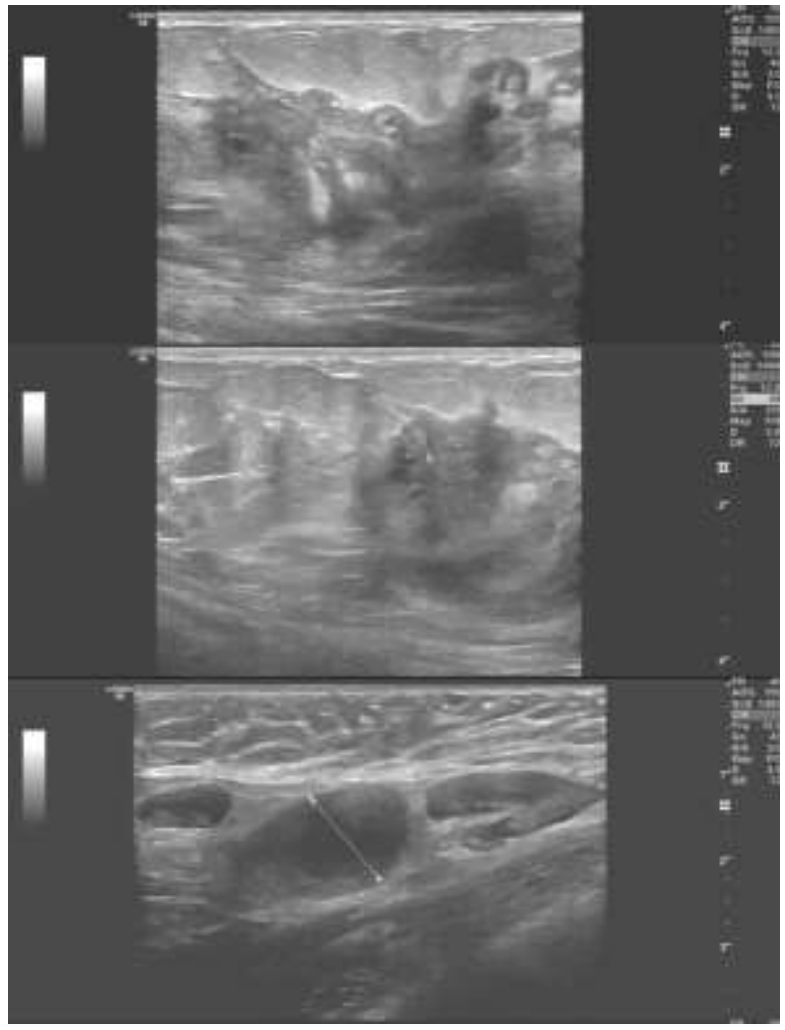

Figure 2b. Widespread infiltrative involvement in both breasts and axillary LAPs are shown.

To evaluate the extent of the involvement, the breast MRI was performed for additional foci. In the mass lesion with smooth lobulated contours in the upper outer quadrant of the left breast, it was noted a mild diffusion restriction in diffusion-weighted sections, increased contrast enhancement from the early arterial phases after intravenous contrast agent injection, showing mild wash out in late phase series (Figure 3).

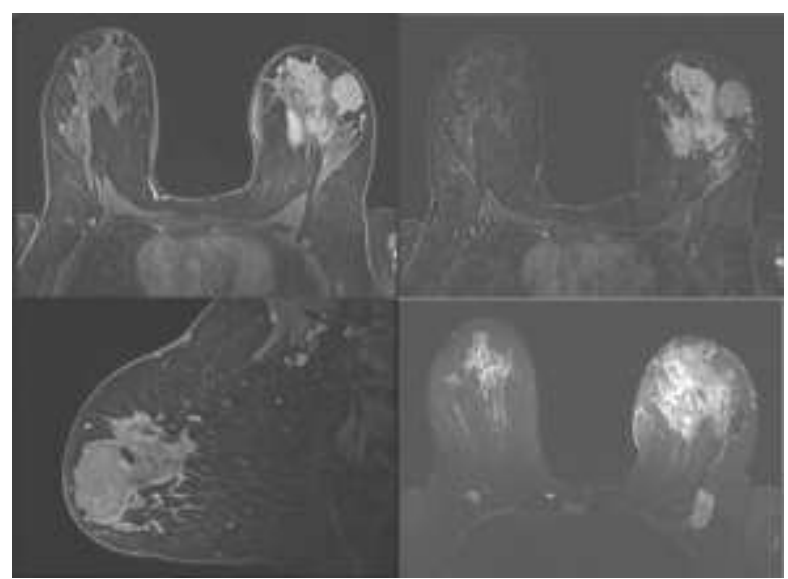

Figure 3. In contrast-enhanced dynamic MRI images of the mass in the upper outer quadrant of the left breast, T1W images taken after intravenous contrast injection show that the mass with smooth contours is homogeneously enhanced, it exhibits a markedly increased heterogeneous enhancement pattern that shows a mass tendency in some places. It is noteworthy that the mass shows mild wash-out in late phase-contrast series and has similar morphological features with axillary LAPs. 
A type 3 enhancement kinetics were detected in the post-contrast dynamic series. Similar nodular lesions of smaller size were observed in both breasts. In addition, increased asymmetric diffuse pathological enhancement in the left breast parenchyma compared to the right, and a lobulated contoured mass with an approximately $4 \mathrm{~cm}$ long axis in the middle inner segment and bilateral axillary multiple pathological LAPs were observed. Core biopsy was performed from the mass lesion in the left breast, and heterogeneous parenchymal areas in both breasts (Figure 4). In immunohistochemical staining, CD20, CD23, BCL2 +, MUM1 + positive; CD5, CD10, BCL6 (-) negative B lymphocytes showing atypical proliferation were detected. The Ki-67 proliferation index was 95\%, C-MYC was $>40 \%$, and the case was evaluated as double-hit bilateral breast lymphoma. The patient was referred to the medical oncology and hematology outpatient clinic without any surgical treatment.

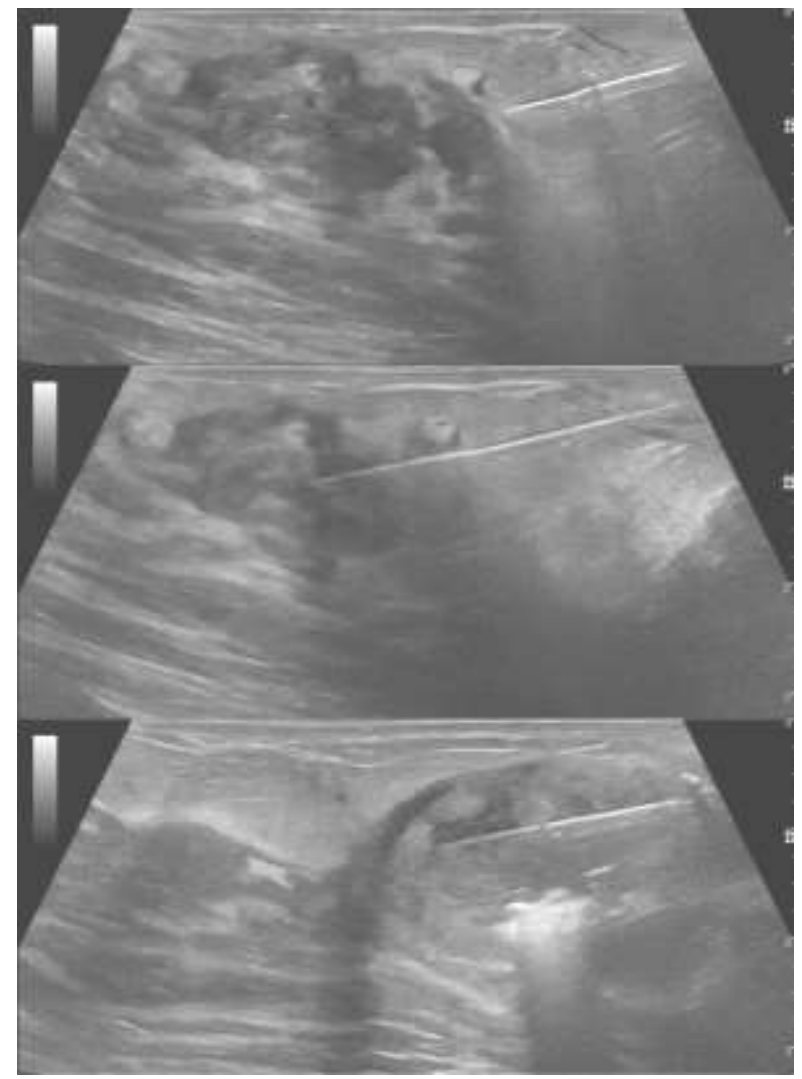

Figure 4. Core biopsy images of the LAP-like mass in the left breast and breast tissue with infiltrative involvement.

\section{Discussions}

Primary breast lymphoma represents about $0.5 \%$ of all malignant breast cancers, while approximately $3 \%$ of extranodal lymphomas are seen in the breast [3,4]. It shows a bimodal age distribution and makes two peaks in the 4th and 7th decades [4]. Generally, diffuse large B-cell nonHodgkin lymphoma is seen, and a small portion of it is associated with breast implants called Breast Implant-
Associated Anaplastic Large Cell Lymphoma (BIAALCL) [3]. The most common type is unilateral involvement which presents as a painless mass. Bilateral involvement is less common and is seen at a younger age [3]. Although our case was 47 years old, it is different in this respect because of bilateral diffuse involvement. The most common mammography finding is mass opacity with smooth contours. The skin edema and thickening are usually associated with high grade. It is seen as a mass lesion with smooth contours showing increased blood supply by color doppler sonography on ultrasonography. It can be isolated or accompanied by patchy heterogeneous areas. In our case, there were widespread heterogeneous hypoechoic areas in ultrasonography, and a smoothly contoured mass.

When the sonographic findings were evaluated in our case, the inflammatory breast cancer was not considered in the patient with bilateral widespread parenchymal involvement because there was no significant effusion in the breast parenchyma or skin. There was no redness, edema or orange-peel appearance on the breast skin. Medullary carcinoma was not considered, because it is mostly a painless soft mass without infiltrative spread. However, in our case, infiltrative spread was detected sonographically in both breasts. Mastitis was not considered, because there was no breast tenderness or redness in the patient without a history of lactation.

Although phyllodes tumor was included among the differential diagnoses in the patient with a rapidly developing painless mass lesion in the left breast, multicentric-multifocal invasive ductal or lobular carcinoma was considered as the main possibility due to bilateral widespread parenchymal infiltrative involvement [4]. However, when neck and inguinal ultrasonography was performed in terms of differential diagnosis of lymphoma in a patient with bilateral axillary diffuse LAPs, similar diffuse LAPs were observed at these levels. For this reason, it was thought that the palpable lesion in the left breast might be a LAP, and the diagnosis was thought as lymphoma with bilateral breast involvement. As a consequence, an MRI was performed to establish extension.

Lymphoma involvements and LAPs are usually isointense in T1 weighted sequences and hyperintense in T2 weighted sequences, generally shows plateau (Type 2) enhancement kinetics on MRI [5]. PET / CT is important in staging. Homogeneous uptake with SUV max value of about 10 is seen. Primary breast lymphoma staging is done according to the Ann Arbor staging system [6]. It is classified as stage 1 if the involvement is limited to the breast, stage 2 if there are more than 2 axillary lymph nodes at the same side with breast involvement, stage 3 if there is lymph node involvement on both sides, and stage 4 if there 
is an extra lymphatic area or organ involvement. Our case was evaluated as stage 4 because of bilateral axillary and breast involvement, as well as widespread involvement in the cervical and inguinal regions [6]. Breast lymphoma is quite different from other breast cancers in terms of treatment. Although the main treatment of breast cancer is surgery, the primary treatment in breast lymphoma is medical. And even if it responds well to medical therapy, the risk of recurrence is high in aggressive forms. Responsible for the poor prognosis, the presence of both the MYC and BCL-2 rearrangements is known as doublehit lymphoma. If accompanied by BCL-6 rearrangement, it is referred to as triple-hit lymphoma. In addition to being a double-hit or triple-hit diffuse large B-cell lymphoma, high $\mathrm{Ki}-67$ proliferation index is also associated with a poor prognosis, as in our case [7].

\section{Conclusions}

In conclusion, breast lymphoma should be considered in the differential diagnosis in middle-aged female patients with a painless soft mass that develops rapidly in the breast, with or without axillary involvement, as well as in patients with bilateral diffuse parenchymal involvement without mass. In this sense, it is important not to perform surgical treatment in patients with lymphoma who do not have a histopathological diagnosis, because the treatment is very specific being essentially a medical / non-surgical therapy.

\section{Conflict of interest disclosure}

There are no known conflicts of interest in the publication of this article. The manuscript was read and approved by all authors.

\section{Compliance with ethical standards}

Any aspect of the work covered in this manuscript has been conducted with the ethical approval of all relevant bodies and that such approvals are acknowledged within the manuscript.

\section{References}

1. Brito-Marcelino A, Duarte-Tavares RJ, Marcelino KB, Silva-Neto JA. Breast cancer and occupational exposures: an integrative review of the literature. $R e v$ Bras Med Trab. 2021;18(4):488-496. doi: 10.47626/1679-4435-2020-595

2. Goh T, Dao K, Rives AF, Fishman MDC, Slanetz PJ. Systemic diseases affecting the breast: Imaging, diagnosis, and management. Clin Imaging. 2021;77:7685. doi: 10.1016/j.clinimag.2021.02.021

3. Franco Pérez F, Lavernia J, Aguiar-Bujanda D, Miramón J, Gumá J, Álvarez R, Gómez-Codina J, Arroyo FG, Llanos M, Marin M, Alfaro J, Quero C, Delgado M, Nogales E, Menarguez F, Martinez N, Torrente M, Royuela A, Abreu D, Provencio M. Primary Breast Lymphoma: Analysis of 55 Cases of the Spanish Lymphoma Oncology Group. Clin Lymphoma Myeloma Leuk. 2017;17(3):186-191. doi: 10.1016/j.clml.2016.09.004

4. El Khoury M, Maietta A, Tran A, Trop I, Lalonde L, Mesurolle B. Case 285: Primary Breast Lymphoma. Radiology. 2021;298(1):231-236. doi: 10.1148/radiol.2020191329

5. Yang WT, Lane DL, Le-Petross HT, Abruzzo LV, Macapinlac HA. Breast lymphoma: imaging findings of 32 tumors in 27 patients. Radiology. 2007; 245(3):692-702. doi: 10.1148/radiol.2452061726

6. Ramadas AA, Jose R, Varma B, Chandy ML. Cervical lymphadenopathy: Unwinding the hidden truth. Dent Res J (Isfahan). 2017;14(1):73-78. doi: 10.4103/17353327.201136

7. Smith SM. Aggressive B-cell lymphoma: the doublehit and double-expressor phenotypes. Clin Adv Hematol Oncol. 2017;15(1):40-42. 